\title{
Die wissenschaftlichen Anfänge von Adolf Meyer (1866-1950) und die Entstehung der «Zürcher psychiatrischen Schule»
}

\author{
Von Hans H. WaLSER, Zürich
}

Es ist nicht einfach, die wirkliche Bedeutung eines großen Arztes für ein Fach der Medizin oder für die Medizin eines Kulturbereiches festzustellen schon gar nicht, wenn es sich um eine jüngstvergangene Periode handelt. Immerhin dürfte Einigkeit darüber bestehen, daß das Lebenswerk von Adolf Meyer für die Grundlegung der heutigen Psychiatrie in den Vereinigten Staaten von entscheidender Bedeutung war. Diese Leistung ist um so imponierender, als er schon zwei Jahre nach seinem Staatsexamen und im Jahre seiner Promotion (1892) in die USA auswanderte, wo er sich großenteils autodidaktisch weiterzubilden hatte. Die Frage nach den persönlichen Voraussetzungen und den wissenschaftlichen Grundlagen, die Adolf Meyer aus Zürich mitbrachte, ist deshalb sicher berechtigt.

Adolf Meyer wuchs in Niederweningen, in einem jener schönen alten Zürcher Pfarrhäuser, heran. Die theologische Herkunft hat ihn sicherlich beeinflußt - Beziehungen zwischen praktischer Theologie und Psychiatrie liegen auf der Hand, jedenfalls wenn die Psychiatrie nicht rein medizinischnaturwissenschaftlich aufgefaßt wird und die Psychotherapie nicht ausschließt. Der Bibliothek des Vaters entnahm er erste psychologische Anregungen, doch war der Vater selbst keine besonders profilierte Persönlichkeit; Meyer rühmt aber die Freiheit seiner Auffassungen und sein undogmatisches Denken auch in theologischen Fragen. Er zeigte wenig Lust, der väterlichen Laufbahn zu folgen, wenn er auch daran dachte, wobei geistesgeschichtliche und philosophische Interessen mitspielten. Wichtiger als berufliches Vorbild war aber der Bruder der Mutter, Dr. Gottraied Walder (1841-1896). Dieser «Onkel Walder», wie Meyer ihn brieflich gelegentlich nannte, war ein sehr gut ausgebildeter, tätiger, angesehener praktischer Arzt. Während seines Medizinstudiums im Zürich der $1860 \mathrm{er}$ Jahre waren Griesinger und Billroth, Horner und Biermer seine Professoren gewesen. Nach der Rückkehr in seine Heimatgemeinde Wetzikon - die Familie Walder stammte wie die Familie Meyer von der Zürcher Landschaft, womit es seine besondere Bewandtnis hat (vgl. 2) - war er auch in öffent- 
lichen Ämtern, als Spitalarzt und in der örtlichen ärztlichen Standesorganisation tätig. Sein Sohn Adolf Walder (1872-1928) setzte die ärztliche Tradition im besten Sinne fort; er wurde Spitalarzt in der nahegelegenen Gemeinde Rüti und vermachte ein beträchtliches Vermögen für die Ausbildung unbemittelter Medizinstudenten. Das Ansehen und die Beliebtheit seines Onkels haben den jungen Adolf Meyer - ganz abgesehen von den spezifischen beruflichen Interessen - beeinflußt, als er als Gymnasiast an die Berufswahl denken mußte. Zudem bestand der Zwang, einen Brotberuf zu ergreifen. Die Stellung der Medizin zwischen Geistes- und Naturwissenschaften, zudem die ethische Verpflichtung, die der Mediziner mit seinem Beruf übernimmt, taten ein übriges. Allerdings strebte Adolf Meyer bald über Vater und Onkel hinaus.

Zur Zeit dieses «Sichfindens» in den oberen Klassen des Zürcher Gymnasiums wurde Adolf Meyer mit Arbeiten des Professors für Psychiatrie, August Forel (1848-1931), bekannt. Daß ein aufgeweckter Gymnasiast in Zürich von Forel hörte, ist nicht verwunderlich, denn der jugendliche, temperamentvolle Westschweizer war schon vor seiner vielumstrittenen, aber populären Agitation gegen den Alkoholmißbrauch weitherum bekannt. Die Umstände seines Amtsantrittes, der erst wenige Jahre zurücklag (1879), waren gar zu auffällig gewesen: Forel hatte - übrigens noch zur Zeit des «Onkel Walder» - in Zürich studiert, sich dann bei Theodor Meynert in Wien und bei Bernhard Gudden in München zum Psychiater ausgebildet und sich in München habilitiert. Als 1879 die Zürcher Professur frei wurde, wäre der erst 31 jährige kaum in Betracht gekommen, wäre nicht an der Universitätsklinik ein Skandal ausgebrochen, der andere Bewerber abschreckte und der auch die Öffentlichkeit in reichlichem Maße beschäftigte. Es hatte damals allgemein beeindruckt, wie rasch und gründlich Forel Ordnung geschaffen hatte und nun einen Lehr- und Forschungsbetrieb aufbaute. Adolf Meyer begeht übrigens einen kleinen, aber bezeichnenden Anachronismus, wenn er schreibt, «noch am Gymnasium» sei er durch «Forels Formulierung und Benutzung der Suggestion und das Verbreiten gesunder Anschauungen im Gebiete der Hypnose » beeinflußt worden - bezeichnend darum, weil wirklich der Hypnotismus wenig später zum Ausgangspunkt einer neuen psychiatrischen Forschungsrichtung und vor allem einer wirklichen Psychotherapie wurde. Forel hat sich aber erst seit 1887, angeregt durch BERnheIm und LiÉBEault, näher mit der Hypnose befaßt - zu einer Zeit also, als Meyer bereits Medizinstudent war. Ohne Vorgeschichte waren Forels Hypnosestudien aber nicht; rückblickend möchte 
man den Hypnotismus als logische Fortsetzung der Anschauungen betrachten, die Forel in den ersten 1880 er Jahren entwickelte.

Am 11. Dezember 1884 hielt Forel einen öffentlichen Vortrag über das Thema: «Das Gedächtnis und seine Abnormitäten». Man möchte denken, daß Adolf Meyer diesen "Rathausvortrag» gekannt hat, den Forel zuerst zwar nicht zu veröffentlichen beabsichtigte, dann aber im folgenden Jahre in gelindem Ärger über eine mißglückte Besprechung in einer Tageszeitung dann doch in Druck gab. Man muß dem wohl etwas überforderten Rezensenten heute dankbar sein, denn dieser Vortrag ist heute ein wichtiger Beleg für den Beginn der "psychologisch-psychotherapeutischen Arbeitsrichtung» bei Forel und damit auch ein Dokument zur Grundlegung der «Zürcher psychiatrischen Schule».

Den schweizerischen Zuhörern von Forels Vortrag mag es nicht weiter aufgefallen sein, für einen in Deutschland ausgebildeten und dort habilitierten Psychiater war es aber ungewöhnlich, daß er sich auf Riвот, einen französischen Autor stützte. Dann folgt der programmatisch gemeinte Satz, daß alle unsere bewußten und unbewußten Seelentätigkeiten im Nervensystem vor sich gehen. Forel versucht dann, das Bewußtsein wissenschaftlich zu erfassen - nun nicht mehr auf irgendeine «hirnmythologische» Weise, sondern mit psychologischer Methodik, wobei er WundT erwähnt. Er unterstreicht, daß die unbewußten Vorgänge «weitaus die Hauptsache, ja fast alles ausmachen». Nicht nur unser Handeln ist weitgehend unbewußt, sondern «wir können auch unbewußt wollen, empfinden und denken». Die bewußten wie die unbewußten Nerventätigkeiten hinterlassen eine «dynamische Spur », ein Erinnerungsbild im Nervensystem, ein Erinnerungsbild, das nicht stabil ist und auf unbewußtem Wege erneuert werden kann. Das Bewußtsein dient «nur als ordnendes, kräftigendes, fixierendes Agens, das mittels der Fähigk eiten des Wiedererkennens, der zeitlichen Lokalisation, der Concentration und der vergleichenden Combination ... viel rascher zu einem vernünftigen, zweckmäßigen Resultate gelangt als die ... unbewußte Tätigkeit». Mit Ewald Hering neigt er dazu, diese dynamische Modifikation der organisierten Materie als etwas allgemeines anzusehen, womit Vererbung und Gedächtnis in nahen Zusammenhang kommen. (Man versteht, daß Forel zwei Jahrzehnte später von Richard Semons «Mneme» so begeistert war!). Im zweiten, klinischen Teil seines Vortrages versucht Forel, Brücken zur somatischen Hirnforschung (insbesondere zur Lokalisationslehre) zu schlagen, wobei er sehr behutsam und kritisch zu Werke geht. Die Amnesien teilt er in organische und funktionelle ein, wobei 
er bei diesen auch den «sogenannten Hypnotismus oder animalen Magnetismus » erwähnt, dessen Studium dann, zwei Jahre später, diese Auffassungen bestätigen und vertiefen sollte.

In diesem Vortrag liegen die wichtigsten Grundzüge von Forels Denken vor - selbst wenn Adolf Meyer ihn nicht gekannt hätte, so kam er mit diesem Gedankengut seit seiner Gymnasiastenzeit in Beziehung. Man erkennt in Forels Ausführungen die werdende Konzeption der «Zürcher Schule», nämlich die ausgewogene Berücksichtigung von psychologischen, hirnanatomischen und allgemein-biologischen Gesichtspunkten, die durch eine solide und kritische klinische Beobachtung und Interpretation zusammengehalten und gewertet werden. Auch die Toleranz und die Offenheit neuen Ansichten gegenüber ist herauszuspüren, wobei für Forel, der sowohl dem französischen wie dem deutschen Kulturbereich zugehörte, die Berücksichtigung auch der französischen Wissenschaft dazukam, was bei der Hypnoselehre entscheidend war.

Daß Forel daran war, der psychiatrischen Wissenschaft neue Horizonte zu eröffnen, muß man auch in seiner Umgebung gespürt haben: Jedenfalls bewarb sich Emil Kraepelin damals um eine Assistentenstelle bei ihm, und es ist in vielerlei Beziehung zu bedauern, daß daraus nichts wurde (zum Briefwechsel Forel-Kraepelin siehe auch 1). Um zu ermessen, was die Einführung des "psychologischen Gesichtspunktes» bedeutete, muß man sich die damalige Lage der Psychiatrie vergegenwärtigen: Noch galt als eigentlich "wissenschaftlich » fast ausschließlich die Hirnforschung, daneben stand die «Irrenpflege» (im weitesten Sinne), die praktisch unbedingt nötig, humanitär unerläßlich, jedoch wissenschaftlich steril war. Wohl bahnte sich allmählich eine psychopathologische Forschung an, aber der damals noch lebende Bernhard Gudden hatte darüber nichts, hingegen viel über Hirnanatomie publiziert. Welche Möglichkeiten aber gerade in dem von den meisten deutschen Psychiatern abgelehnten Hypnotismus lagen, sollte etwas später Sigmund Freud beweisen. Sogar in diesem frühen Vortrag Forels fallen Ähnlichkeiten mit dem Freud der frühen analytischen Periode auf, so die hohe Schätzung der «unbewußten Prozesse» und die «dynamische» Auffassung - so verschieden die weitere Ausgestaltung auch gewesen sein mag.

Als Adolf Meyer in diese Zusammenhänge eintrat, hat er deren Hintergründe gewiß noch nicht überblicken können. Aber er spürte, daß sich hier fruchtbare Forschungsgebiete auftaten. So schreibt er später, in Erinnerung an jene Zeit (10): 
« Es bestand damals wie auch noch jetzt ... der Zwang, immer gleich die Tatsachen, die man am besten kennen lernen könnte, so wie sie uns vorkommen, entweder in neurologischen oder endokrin-physiologisch-chemischen Formeln auszudrücken ... Lebensereignisse, die man sehr wohl mit unseren tagtäglichen Ansichten beherrschen und verständlich machen könnte, sollten keinen wissenschaftlichen Wert haben, wenn dafür nicht eine gehirnanatomische oder endokrin-physiologische Formel gefunden war ... So kam es denn, daß, was psychologisch war, als von der Natur ausgeschlossen betrachtet wurde, es sei denn, daß man damit auf das Gehirn verweisen konnte ... Mit Forels Auffassung der Hypnose gestaltete sich tatsächlich eine freie physiologische und doch psychologische und recht menschlich lebendige Auffassung der Vorgänge.»

Etwas weiteres wird in Forels Vortrag deutlich: er hatte immer das Bestreben, auch die psychischen Erscheinungen im biologischen Gesamtzusammenhang allen Lebens zu sehen. Das führte ihn ja später auch zum Monismus. Für Meyers Werk wurde der Begriff «Psychobiologie» geradezu zu einem Schlagwort, und wenn diese dann für ihn doch anders aussah, so war mit Forel doch der Ansatz im Biologischen gegeben.

Den jungen, ideal gesinnten Mediziner muß es schließlich beeindruckt haben, wie sehr Forel am Schicksal seiner Patienten menschlich Anteil nahm. Auch das war in der damaligen Psychiatrie keine Selbstverständlichkeit - denn «wissenschaftlich» ließ sich damit ja nichts anfangen. Es ist auffällig, daß Forels therapeutische (und auch gewisse erkenntnismäßige) Fortschritte auf Schicksale einzelner Patienten zurückgehen, handle es sich nun um den Somnambulismus (bzw: die Hypnose), um die Arbeitstherapie, um die Strafrechtsreform in psychiatrischer Sicht oder um die Behandlung der Alkoholkrankheit. Ein wichtiger Prüfstein dafür, was ein Arzt vom anderen als Mensch und Therapeut wirklich denkt, liegt darin, ob er ihm sich selbst oder nahe Angehörige zur Behandlung anvertraut - und Adolf Meyer war sichtlich froh, als er seine Mutter bei einer depressiven Erkrankung in der Behandlung Forels wußte (Brief an Forel vom 3. Januar 1893; vgl. auch 9, S. 132 f., und 13).

Nun war August Forel aber auch psychiatrischer Wissenschafter im Sinne der Zeit, nämlich Hirnanatom - anders hätte er sich ja auch den Weg zur akademischen Laufbahn kaum öffnen können. Die erste Bekanntschaft mit der Hirnanatomie hatte Adolf Meyer 1886 bei Constantin von Monakow gemacht, doch wurde er von den Arbeiten Forels besonders beeindruckt, so zunächst von derjenigen aus dem Jahre 1887 (5), in welcher die Grundsätze der (späteren) Neuronenlehre entwickelt wurden, dann aber auch von Forels Habilitationsschrift (6) aus dem Jahre 1877 über die «Haubenregion » (tegmentum) im Gehirn. Meyer schrieb später darüber: 
"I am perhaps too enthusiastic about Forel's paper on the tegmentum. Yet I believe it is to this paper that I owe nearly all my education for anatomical studies."

Und man begreift sein Vergnügen, als er sich nach Theodor Meynerts Tod dessen annotiertes Exemplar dieser Schrift verschaffen konnte - gerade weil Forel darin seinem früheren Lehrer widersprach. So schrieb Adolf Meyer am 10. August 1892 an August Forel:

«Der eigentliche Grund meines Schreibens ist aber etwas anderes. Ich habe durch einen Freund aus Meynert's Nachlaß unter anderen Sachen auch Ihre Haubenarbeit bekommen. Es ist zu fröhlich, wie aufmerksam er dieselbe durchstudiert. Meynert hat, den Blau- und Rotstift in der Hand, eine Anzahl von Zeichen der Approbation und, gelegentlich ${ }^{\text {* auch }}$ des Widerspruchs und zahlreiche Bemerkungen angebracht, die das Buch, das ja unter allen mir zugänglich gewesenen hirnanatomischen Arbeiten schon den ersten Rang einnimmt, ganz unbezahlbar machen. Wenn ich wieder einmal nach Zürich kommen werde, sollen Sie das Prachtsstück sehen. Meynert hat gewiß nicht manches Buch so aufmerksam durchstudiert wie dieses. Auch die «Gehirnanatomischen Betrachtungen» von 1887 hat er aus Leibeskräften gewürdigt.»

Adolf Meyer kam übrigens nicht mit Selbstverständlichkeit zu einer psychiatrisch-neurologischen Laufbahn, sondern er dachte zunächst an die innere Medizin, und insbesondere an das Doktorieren und an eine Assistentenstelle bei dem Zürcher Kliniker Hermann Eichнorst. Das lag für einen jungen, strebsamen Mediziner nahe, da ja die innere Medizin sehr viel von der neuen, «naturwissenschaftlichen» Methodik profitiert hatte und als besonders fortschrittliches Fach galt - im Gegensatz zur Psychiatrie, wie sie vielerorts gelehrt wurde. Da war es nun - neben einer Verstimmung mit Eichhorst - doch gerade die Tatsache, daß Meyer in Zürich von der Psychiatrie «ein überaus eindrucksvolles Bild mit großen Forschungsmöglichkeiten » bekam, die den Ausschlag für seine weitere Laufbahn gab. Im Jahre 1893 hat Adolf Meyer selbst darauf hingewiesen, wie viel Hirnforschung damals - auch außerhalb von Forels Klinik - getrieben wurde (10). Von den in diesem Zusammenhang genannten Männern (u.a. PAuL Martin, J.Gaule, R. Wlassak, G.Huguenin) hat ihn aber, außer C. von Monakow, wohl nur der sonst vergessene J.J.Honegger beeinflußt, den er in seiner Dissertation wiederholt nennt.

Die persönlichen Beziehungen zwischen August Forel und Adolf Meyer begannen - wie diejenigen zwischen Forel und Oscar Vogt (4) - nicht gerade vielversprechend. Der angehende Doktorand hatte zuerst einmal eine Standrede des Professors über sich ergehen zu lassen, der über die Kandidaten schimpfte, die dem Doktorvater die Beendigung der angefangenen Ar- 
beit überlassen. Dann erhielt Meyer aber doch fürs erste ein bereits geschnittenes Chamäleongehirn zur Bearbeitung. Forel überzeugte sich bald von den wissenschaftlichen Qualitäten und vom guten Charakter seines Doktoranden. Das freundschaftliche Verhältnis, das damals entstand, sollte lebenslangen Bestand haben. Gerade die Briefe Adolf Meyers, die sich im Besitze des Zürcher medizinhistorischen Institutes befinden, lassen erkennen, daß ihm Forel mehr war als ein bloßer wissenschaftlicher Mentor. Man wundert sich nicht, wenn er, Jahrzehnte später, den Gratulationsartikel zu Forels 80. Geburtstag (1928) mit den Worten einleitet:

«Neben den Wechselbeziehungen zwischen Eltern und Kind ist wohl das Verhältnis zwischen Lehrer und Schüler das potentiell wichtigste in der Entwicklung einer Zivilisation und für die individuelle und gruppenmäßige Teilnahme am Strome des Lebens.»

Meyer hat sich hier gewiß richtig erinnert: August Forel war für ihn in seiner «Zürcher Zeit» ein väterliches Vorbild, ein Mann, der ihm an persönlicher und wissenschaftlicher Statur das bieten konnte, was er bei Vater und Onkel, die seinen frühen Interessen die Richtung wiesen, nicht mehr hatte finden können. Am 28. Dezember 1893 schrieb er aus Kankakee (Illinois, U SA), wo er an einer psychiatrischen Klinik tätig war, an seinen früheren Lehrer u.a.:

«Ich wünsche aufrichtig, ich hätte Ihre Klinik mit mehr Verständnis verfolgt und auch länger besucht. Ein Semester und einige Kliniken später, als ich im Burghölzli arbeitete, das ist alles, was ich an Psychiatrie mit nach Amerika gebracht habe. Ich muß sagen, es zeigt sich jetzt, daß es eine gute Grundlage war, und ich hoffe mit der Zeit so weit zu kommen, daß ich mich Ihr Schüler nennen darf, ohne daß Sie sich gegen solche Anmaßung verwahren müßten. Ist auch von dem Detail manches verschwunden und ganz dem Gedächtnis entfallen, so ist doch von dem Geist der Auffassung und der Methode manches hangen geblieben.»

Adolf Meyer sagt hier deutlich - wenn auch mit bezeichnender Bescheidenheit - daß er sich als Schüler Forels betrachtet. Aber man darf dabei keinesfalls übersehen, daß Meyer schon früh eine große Selbständigkeit und Zielstrebigkeit an den Tag legte. Man kann etwa daran erinnern, daß er am Ende seines Studiums Anregungen vor allem in Paris, Edinburg und London suchte - statt in Wien oder Berlin, wie das bei den Zürcher Studenten damals wie schon zur Zeit seines Onkels üblich war. Und gerade diese Studienaufenthalte im französischen und im englischen Sprachgebiet wurden für ihn entscheidend wichtig (vgl. insbesondere 10,12). Eindrücklich ist vollends, wie sich der kaum Promovierte einer wissenschaftlichen Laufbahn zuwandte und dazu auch gleich in die Vereinigten Staaten auswanderte. Daß der persönliche Einfluß Forels - so wichtig er auch sein mochte - ein 
gewisses Maß nicht überschritt, läßt sich an Meyers Einstellung zur Alkoholabstinenz ablesen. Für Forel war die totale Abstinenz ein ethisches Postulat, das er mit aller Kraft auch unter seinen Schülern verbreitete. Gewiß wurde auch Meyer davon beeinflußt - ohne sich aber zur vollen Abstinenz zu verpflichten. Er scheint es Forel hoch angerechnet zu haben, daß er ihm trotzdem seine Freundschaft bewahrte, und fand dafür die hübsche Formulierung:

"I never became a militant total abstainer, as Forel would have liked. Yet he respected my independence."

Überhaupt scheint Forels Art Meyers Unabhängigkeitsbedürfnis eher angespornt zu haben.

Aus der oben zitierten Briefstelle ist weiter ersichtlich, daß Adolf Meyer schon kurz nach seiner Auswanderung sah, daß er weniger Einzelheiten der Forel-Schule, als Grundlagen methodischer und auffassungsmäßiger Art übernommen hatte, auf denen er dann sein eigenes Gebäude errichtete. Gewisse Gemeinsamkeiten bezüglich späterer Arbeitsgebiete sind aber auffällig wobei freilich schwer auseinanderzuhalten ist, wieviel auf das Konto des «wissenschaftlichen Zeitgeistes» geht und wieviel einer originalen Entwicklungslinie zugehört. Forel wie Meyer gingen von der Hirnforschung aus, wandten sich aber später mehr der klinischen Psychiatrie, der Psychopathologie und «psychiatrisch-sozialen» Fragen zu - Forel mit seinen vielen «außermedizinischen» Interessen sogar noch in ausgeprägterem Maße. Beide stießen so auf die Wichtigkeit der Prophylaxe, also der seelischen Hygiene. August Forels Buch Hygiene der Nerven und des Geistes im gesunden und kranken Zustand erschien erstmals 1903. Und wenn Adolf Meyer im Jahre 1908 von dem Laien CLIFFord W. BeErs Anregungen zum seelischen Gesundheitsschutz übernahm - nicht ohne dessen «zuerst noch etwas gereizten Eifer gegen die Anstalten» ins Konstruktive umzubiegen - so denkt man unwillkürlich an die Vorurteilslosigkeit, mit welcher Forel 1884 vom Schuhmacher Јаков Bоssнавдт eine wirksame Behandlung der Alkoholkrankheit «erlernte». Auch an die akademische Lehrtätigkeit ist zu erinnern: Wenn Adolf Meyer allerdings auf diesem Gebiete ins Große wirken konnte, während August Forel mehr wie ein «Rufer in der Wüste» erscheint, so liegt das an der Verschiedenheit der Verhältnisse in den weiträumigen, im wissenschaftlichen Aufbau begriffenen Vereinigten Staaten verglichen mit denjenigen in einem europäischen Kleinstaat. Beide Männer empfanden es aber als dringendes Anliegen, zunächst dem Psychiater, aber 
auch dem praktischen Arzt eine möglichst gute Ausbildung besonders auch in medizinischer Psychologie zu geben.

Im vorliegenden Aufsatz sollte darauf hingewiesen werden, wie wichtig für die wissenschaftliche Entwicklung von Adolf Meyer - und damit auch für das Schicksal der amerikanischen Psychiatrie in den letzten Jahrzehnten - die «Lehrjahre» dieses bedeutenden Psychiaters an der Zürcher Universität und im Laboratorium der dortigen psychiatrischen Klinik waren. In jenen Jahren entstand - insbesondere durch August Forels originelle Konzeptionen - die «Zürcher psychiatrische Schule». Adolf Meyer erhielt so einen großen Eindruck von den Zukunftsaufgaben und den Forschungsmöglichkeiten dieses medizinischen Gebietes.

Eine besondere Freude ist es für uns, diese Arbeit E.H.Ackerknecht zum 60. Geburtstag zu widmen, nicht nur, weil sie sich auf unveröffentlichte Briefe stützt, die sich im Besitze des von ihm geleiteten Institutes befinden, sondern auch, weil er Adolf Meyer noch selbst in Baltimore (U S A) kennengelernt hat und gelegentlich von der sympathischen Persönlichkeit dieses aus der Zürcher Landschaft stammenden Arztes erzählte, der für die psychiatrische Wissenschaft in den Vereinigten Staaten eine so große Bedeutung erlangte.

\section{Literatur}

1. AcKerknecht E.H., Ein Brief Emil Kraepelins an Auguste Forel. Festschrift zum 60.Geburtstag von Manfred Bleuler, o. O. u. J. (1963).

2. Bleuler Manfred, Early Swiss Sources of Adolf Meyer's Concepts, Amer. J. Psychiatry, Band 119, Nr.3, September 1962.

3. Diethelm Oskar, Adolf Meyer, in Kurt Kolle, Große Nervenärzte, Bd. 2, Stuttgart 1959.

4. Forel August, Rückblick auf mein Leben, 2. Auflage, Zürich 1947.

5. Forel August, Einige hirnanatomische Betrachtungen und Ergebnisse, Arch.Psychiatrie, Band XVIII, 1887.

6. Forel August, Untersuchungen über die Haubenregion und ihre oberen Verknüpfungen im Gehirne..., Arch. Psychiatrie, Band VII (1877).

7. LiEf A., The Commonsense Psychiatry of Adolf Meyer, New York/Toronto/London 1948.

8. Meyer Adolf, Correspondenz-Blatt für Schweizer Ärzte XXIII (1893) 202, 245 (mit Nachwort von A. ForeL).

9. Meyer Adolf, Psychobiology a Science of Man, Springfield (Illinois, U SA) 1957.

10. Winters Eunice E. (General Editor), The Collected Papers of Adolf Meyer, 4 Bände, Baltimore 1950-1952.

11. Archives of Neurology and Psychiatry, Band 37, Nr.4, April 1937 (Special Number dedicated to Dr. Adolf Meyer).

12. Supplement to Bulletin of the Johns Hopkins Hospital, 89 (Juli 1951) Nr. 1 (Adolf Meyer Memorial).

13. WaLser H., August Forel Briefwechsel (in Vorbereitung). 